\title{
Brief Introduction to the History of Burns Medical Science
}

Fire was, perhaps, man's first double-edged sword, for, throughout history, it has both served and destroyed mankind. While fire served to keep wild animals at bay in the night and warm people chilled by the winter air, it also turned on its master. From time to unfortunate time, fire leapt out at man and caused what remains today one of the most painful of human experiences, the burn.

Burns injuries were first described in the Ebers papyrus (1500 B.C.) which tells the reader that a delicate mixture of cattle dung and black mud was 'just what the doctor ordered' for a burn. Through centuries that followed any physician worthy of note had a favorite remedy for the relief of burns pain and suffering. Dupuytren, the famous 19th century French surgeon who first described the contracture that bears his name wrote: 'Burns had been the object of one of the most bizarre treatment methods'. Fabricius Hildanus, a 15th century German physician, was the first to classify burns into three degrees and debates raged well into the 20th century about how best to treat the burns - to cool or to not cool, to moisten and drain or to dry and seal for sterility. Finally, consensus was reached after the First World War that the best treatment for burns was surgical skin transplantation with subsequent scar reduction and pain control medications as needed. In the early 1950s, spurred on by thermal injuries during the Korean War, the US government established the original Surgical Research Unit (The US Army Burn Center) at Brooke Army Hospital in San Antonio, Tex., USA where skin grafting became the preferred treatment for $30 \%$ total body surface area (TBSA) burns. Survival was now the expected prognosis and one counted oneself lucky to survive.

Since the 1950s and 1960s, many medical experts from other countries threw themselves into the research work of burns medical science and contributed a great amount of experimental data which advanced the field of burns treatment. By now, patients with more than $90 \%$ TBSA burns can expect a fighting chance for survival when of- fered treatment from a protocol involving surgical burns therapy consisting of localized treatment and systemic medical management. Once established in academic teaching centers, this two-pronged approach was quickly practiced around the world. The localized treatment of the 1960s was typified by a drying of the burned skin which enabled a crust (deep, partial-thickness) or eschar (full-thickness) to develop over the burned tissue. This crusting was accompanied by surgical excision of necrotic skin tissues and of viable dermis (tangential excision of crust). In addition, whole subcutaneous tissue (fascial debridement of eschar) was also an all too frequent aspect of the treatment. After this debridement was achieved, autografts or cultured epithelial autografts were placed on top of the lesion to close the wound from exogenous infectious agents. In the case of small, deep burns, initial excision and immediate autografts were recommended in the early stage after an injury. The systemic treatment, based upon what was then known about burns pathophysiology, was practiced in accordance with conventional surgical wounds management. This combined therapy consisted of medical management to avoid shock syndrome as well as to avoid infection while at the same time offering local and systemic nutrition support for tissue and whole body physiology, respectively. A great many protocol formulas were championed by leading scientists and doctors and these were offered with qualified success worldwide. This treatment became the 'standard of care' and became known collectively as 'conventional surgical burns therapy' or 'surgical excision and skin grafting burns therapy'. Its theories and treatment measures were compiled in medical textbooks worldwide prior to being introduced into China in the late 1950s. A recent improvement of this conventional surgical therapy was the innovation by American doctors who successfully treated patients with extensive, deep burns by using cultured composite autografts. This represented an important advance in the autograft technique. 
In the 1980s, burns specialists began to look deeper into the physiology of traumatic burns wounds responding to conventional therapies. To their chagrin, these burns specialists discovered that these 'state-of-theart' clinical treatment protocols, while representing a lifesaving improvement compared to the primitive pre1940s protocols, nonetheless remained a merely destructive therapy as far as the localized tissue was concerned. These burns specialists noted that conventional therapies neither rehabilitate the burned tissue itself, nor do they cooperate with the natural physiological repair mechanisms of burned tissue. Therefore, the feasibility and reasonableness of conventional surgical therapy, characterized as it is by dryness, excision and grafting, was evaluated and found lacking both in theory and methodology. Although Western researchers conducted massive experimental studies that addressed concerns of desiccation, excision and skin grafting, little progress was attained and ultimately the clinician was left with a suboptimal medical result - the disfiguring scar. This arena of painful dressing changes, rampant infection, devitalized tissue and residual scarring was the frustrating stage upon which the burns therapist pleaded for innovation but upon which no champions advanced until recently.

During World War II, an alert and observant Army surgeon, Joseph E. Murray (born April 1, 1919), had noted that skin grafts were only compatible between identical twins. From this observation, Murray then postulated that transplantation of internal organs might also be fraught with rejection and he began the experimentation, initially with canine and later with human kidneys, which ultimately resulted in his sharing the 1990 Nobel Prize for Physiology or Medicine with E. Donnall Thomas. Murray's work in organ- and tissue-transplant techniques set the tone for burns therapies for the rest of the 20th century. Consistent with the reductionistic genius of the American mind, an ill patient was seen as a collection of parts some functioning better than others. In the case of the burns patient, the therapeutic goal became to surgically remove the burned parts before transplanting thereupon some unburned parts. It was no surprise that, prior to Murray and Thomas, the host system rejected the graft tissue since a living being is far more than the sum of its parts. Today, potent immunosuppressive pharmaceutical agents are required for successful transplantation protocols in burns. Though life-saving, these drugs, true to their name, hobble the native host immune system of the surviving burns patient. Frequently, the doctor is chagrined at the trade-off whereby his patient survives - but at the expense of his immune system. As in most areas of medicine and surgery, burns specialists suffered along with their patients for they knew that there must be a better way to help those burned patients.
Nonetheless, despite the frustrating situation where the best the burns specialist could offer would be a life hobbled by chronic pain and disfiguring and motionrestriction scarring topped by systemic immunosuppression, no one was 'thinking outside of the box'. Beneath this consensus that transplantation surgery was the treatment of choice, we can now discover another unspoken consensus, i.e. that burns are a disease of the skin and therefore ought to be treated dermatologically rather than systemically or holistically. Everyone saw that the burned part was the problem and that it should be replaced.

In the 1970s, in China, Professor Xu Rong Xiang alone was thinking outside of the box where he boldly established an entirely new theory of burns physiology upon which he then built a dramatically effective burns treatment which he called 'Burns Regenerative Therapy' (BRT). This innovation, which integrates moist-exposed burns treatment (MEBT) and moist-exposed burns ointment (MEBO), was a balm to the struggling burns therapy industry. The therapeutic essence of MEBT/MEBO is to maintain the burns wound in an optimum physiologically moist environment through the use of a specially designed ointment - MEBO. Rather than surgically excising the burned tissue and its underlying dermis, the goal became to heal the burned tissue and stack the cards in favor of tissue regeneration - an unimagined goal. MEBO, the patented topical remedy, is composed of natural plant extracts dissolved in a sterile and refined sesame-oil base with beeswax as a preservative. When applied topically, MEBO promotes burns tissue repair in an astonishingly effective manner. Initially, MEBO cleans the burned tissue by stimulating the discharge and removal of debris (liquefaction of necrotic tissues). As a complementary healing benefit, MEBO also enhances the regeneration and repair of the residual viable tissue at the base and periphery of the burn in order to anchor vitality within the wound-healing process. Coincident with the application of MEBO, a systemic comprehensive treatment is initiated based on the natural pathophysiology of burned tissue. Accordingly, BRT and MEBT/MEBO is distinguished from conventional surgical therapy in that dryness, excision, skin grafting and scarring as well as the excruciating pain associated with dressing changes is no longer a necessary component of burns care.

The history of MEBT/MEBO is quite auspicious and parallels the ascendancy of China in the marketplace of modern times. Today, the West embraces China as one of the three countries in the history of mankind which were able to safely send a man into space. Equally so, Western doctors who have observed the miracle regenerative cures of MEBT/MEBO embrace Dr. Xu and his team as pioneers in burns therapies. The West first learned about MEBT/MEBO on August 16, 1988 via a Chinese press release that declared the clinical success of this newly dis- 
covered burns treatment theory and its uniquely efficacious therapy. Bolstered not only by clinical success (both in China and abroad) but also supported by copious scientific research, MEBT/MEBO immediately altered the direction of academic research in burns treatment worldwide.

$\mathrm{Dr} . \mathrm{Xu}$ is one of the bright lights in the firmament of scientists alive today. Yet he too stands above the shoulders of scientists who came before him. The treatment philosophies of traditional Chinese medicine urge the pursuit of regeneration as opposed to replacement of burned or diseased tissues as have a precious few Western doctors who sought to apply agents to improve and accelerate the wound-healing process. Ambroise Pare (1510$1590)$ postulated that a surgeon's goal in wound management was to create an environment where the healing process could proceed in an optimal fashion. Pare demonstrated the beneficial effect of the application of hot oil to fresh open wounds. Since then and over the centuries many publications have pointed out that a moist environment enhances epithelialization in the wound-healing process. Controlled experimental and clinical data have in recent times supported the suggestion that a moist environment enhances wound healing in the form of an occlusive dressing compared with a dry environment. Xu has developed MEBT - a therapeutic procedure based on the moist environment of the wound, using an ointment that enhances epithelial repair, and in particular that of partial-thickness burns wounds. MEBO consists only of natural ingredients including - apart from honey and sesame oil -17 amino acids, 14 fatty acids, and 4 polysaccharides. The ointment's main active substance is considered to be $\beta$-sitosterol at a concentration of $0.25 \%$. Clinical and experimental investigations by Chuanji, Yunying and $\mathrm{Xu}$ have indicated that MEBO has the following therapeutic effects:

1 Analgesic: MEBO reduces pain in partial-thickness burns wounds.

2 Anti-shock: MEBO reduces evaporation of water from the burns wound surface and improves microcirculation by decreasing peripheral and systemic capillary exudation.

3 Anti-bacterial: MEBO changes the biological behavior of bacteria, inducing a decrease in bacterial toxicity and invasive capacity, as well as sensitivity to antibiotics; it also increases the wound's local and systemic immunity.
4 MEBO promotes epithelial repair; it also reduces healing time in partial-thickness burns.

5 MEBO improves and reduces scar formation and contributes to the formation of a smooth, thin, and aesthetically acceptable scar, thus preventing the formation of hypertrophic scars.

In 1989, Americans finally learned that the paradigm had shifted in burn care when Newsweek published a report subtitled: 'Could a new medication from China change the world's approach to treating burn injuries?' This caught many US doctors unawares and even today, 14 years later, $90 \%$ of US burns specialists are unaware that this BRT and MEBT/MEBO has been validated in hundreds of experimental studies and clinical practices around the world. These results substantiate the claim that the theory and practice of BRT and MEBT/MEBO comprise a successful revolution in burns care by offering a patently superior methodology of burns treatment when compared to the desiccation, excision and grafting required by conventional therapy. In addition, BRT and MEBT/MEBO also offered the first sophisticated and accurate characterization of natural burns pathogenesis, allowing scientists around the world to finally understand the principles of effective therapeutic burns treatment. MEBT/MEBO therefore attained the rarified status of a truly revolutionary and beneficial clinical success story. With this new therapy, which heralds an advancement into a new field of burns medical science, patients sustaining partial-thickness or full-thickness dermis burns cannot only survive what once were life-threatening burns injuries, but can now do so without inordinate pain, immune-depleting surgical excision or the disfiguring scars from the now obsolete surgical technique of skin grafting. Today, the history of burns therapy has advanced into a bright and promising future. Professor $\mathrm{Xu}$ is teaching the world to work with the regenerative forces of nature. In the pages that follow, Professor Xu welcomes collaboration as we surge forward together committed to reducing the pain, disfigurement and suffering of burns patients the world over. Let us strive together for this noble and finally attainable goal.

Bradford S. Weeks, MD 